\title{
Facilitation of schedule-induced behavior
}

\author{
TIMOTHY O. SHEARON and JOSEPH D. ALLEN \\ University of Georgia, Athens, Georgia
}

\begin{abstract}
Sixteen food-deprived Norway rats were run on a standard schedule-induced polydipsia training schedule until drinking was stable. Subjects were then assigned randomly to receive a 5-day, 2-week, 4-week, or 10-week delay before reacquiring polydipsia. No differences were obtained for milliliter consumed during acquisition or reaquisition except for an increase in milliliter consumed for the 5-day delay group during reacquisition. The implications of this result are interpreted in light of expected results.
\end{abstract}

Falk (1961) demonstrated that food-deprived rats consumed excessive quantities of water when they were given food on a schedule. This excessive drinking was characterized as occurring postpellet and, at that time, unexpectedly regularly. This phenomenon was labeled schedule-induced polydipsia (SIP) (Falk, 1969). SIP has since been obtained with many schedule parameters assigning either response-dependent (e.g., fixed-interval schedules) or response-independent [e.g., fixed-time (FT) schedules] reinforcers (Segal, 1965). SIP has been demonstrated in several species, including pigeons (Shanab \& Peterson, 1969), humans (Kachanoff, Leveille, McLelland, \& Wayner, 1973), monkeys (Porter $\&$ Kenshalo, 1974), and gerbils (Porter \& Bryant, 1978).

Further research has shown SIP to be one of a group of behaviors subsequently referred to as scheduleinduced behaviors (Falk, 1971; Staddon, 1977). Schedule-induced behaviors are defined as behaviors, other than those targeted by the reinforcer, that are facilitated by the presentation of reinforcers on a schedule (Staddon, 1977). Additionally, scheduleinduced behavior is characterized by two pervasive qualities: It is excessive and extremely persistent (Falk, 1971). One area that has received little research is the acquisition or reacquisition to steady-state performance of SIP (by reacquisition is meant the recapturing of steady-state SIP after, e.g., behavioral, temporal, or neurological intervention). The present study investigated the robustness of SIP across various delays to reacquisition.

\section{METHOD}

\section{Subjects}

Sixteen 90-day-old male hooded Norway rats obtained from the colony at the University of Georgia served as the subjects for the study. The subjects were maintained at $80 \%$ of their free-

Portions of this research were presented at the 1984 meeting of the Eastern Psychological Association in Baltimore, MD. Requests for reprints should be sent to J. D. Allen, Department of Psychology, University of Georgia, Athens, GA 30602.

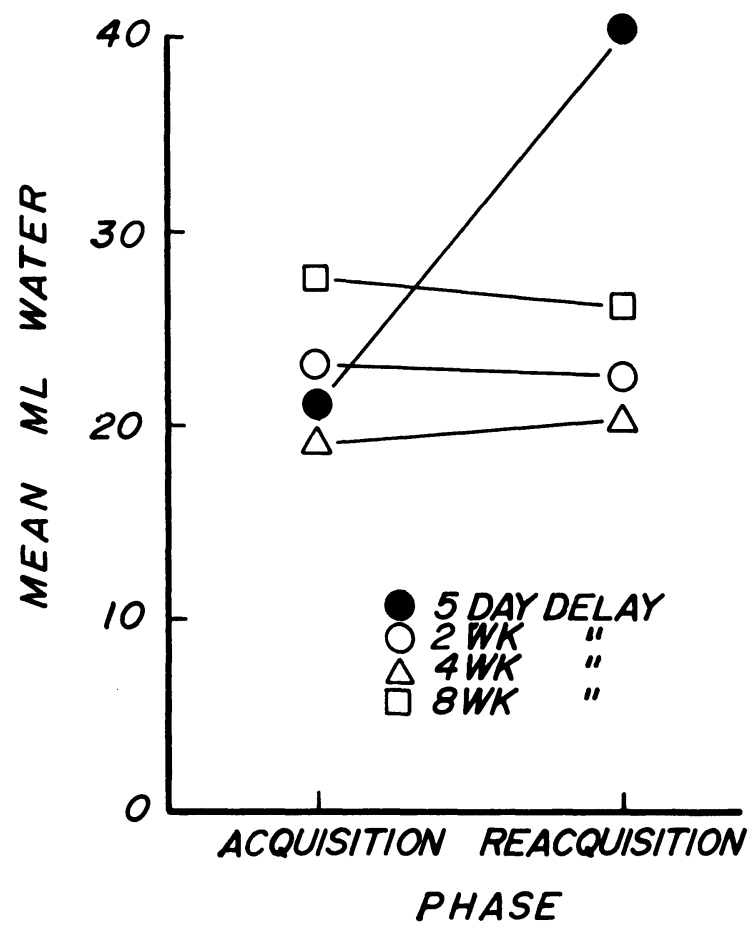

Figure 1. Stable group means for acquisition and reacquisition in milliliter of water consumed per session for the 5-day, 2-week, 4-week, and 10-week groups.

feeding weights by limited access to Purina Lab Chow and unlimited access to water in the home cage.

\section{Procedure}

The subjects were assigned to one of the following conditions (four subjects per condition): 5-day, 2-week, 4-week, or 10-week delay to reacquisition. Sessions were $1 \mathrm{~h} /$ day, 7 days/week. For baseline, 6045 -mg Noyes pellets were placed in the food magazine prior to the session, and drinking was recorded after $60 \mathrm{~min}$. SIP training sessions consisted of 60 presentations of a single $45-\mathrm{mg}$ Noyes pellet on a FT 60 -sec schedule. Acquisition was run until water consumption was stable, stability being defined as 5 days with drinking varying (plus or minus) no more than $5 \mathrm{ml}$ from the mean of those 5 days. Each group was then given a delay before reacquisition of SIP of from 5 days to 10 weeks, based on group assignment. During reacquisition, the standard SIP procedure was followed under the same parameters as in 
initial acquisition (i.e., 60 presentations of a single pellet on a FT 60-sec schedule).

\section{RESULTS AND DISCUSSION}

Figure 1 presents the group means for milliliters of water consumed across both acquisition and reacquisition. The only differences in milliliters consumed occurred in reacquisition. These differences were obtained in the reacquisition for the 5-day group, which was originally intended to be the control group. Because these results lie in the realm of anomalous findings, statistical analyses were not performed on the data. Confidence

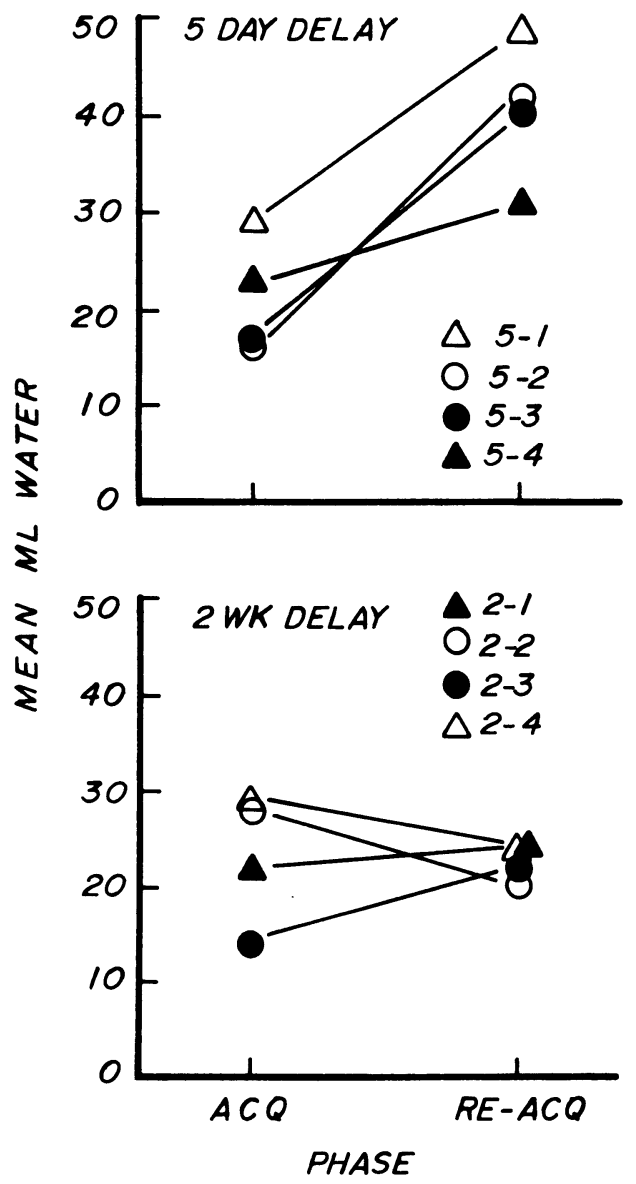

Figure 2. Stable means in milliliter of water consumed per session for the individual subjects in the 5-day and 2-week groups during acquisition and reacquisition phases. in the results is increased somewhat by the data for individual subjects. Figure 2 presents individual means for milliliters of water consumed across acquisition for the 5-day and the 2-week delay groups. Examination of Figure 2 reveals that all five subjects in the 5-day delay group increased the milliliters consumed during reacquisition. The individual data for the 2-week group were chosen arbitrarily. These data are consistent with the data from the 4- and the 10-week delay groups in that in no more than two cases per group was any increase in milliliters consumed noted during reacquisition. In addition, in no case was the milliliter increase of these animals equal to the smallest increase in the 5-day group.

The results of this study show, first of all, that SIP is an extremely robust phenomenon, since there was no apparent relearning or readaptation necessary after a delay-to-reacquisition exceeding 2 months. Second, it seems that SIP, although already characterized by its excessiveness, may be manipulable to even greater extremes than previously obtained. Further research into the replicability of this finding and the range of parameters under which it is obtainable is being carried out.

\section{REFERENCES}

FalK, J. R. (1961). Production of polydipsia in normal rats by an intermittent food schedule. Science, 133, 195-196.

FALK, J. R. (1969). Conditions producing psychogenic polydipsia in animals. Annals of the New York Academy of Sciences, 157, 569-593.

F ALK, J. R. (1971). The nature and determinants of adjunctive behavior. Physiology \& Behavior, 6, 577-588.

Kachanoff, R., Leveille, R., McLelland, J. P., \& Wayner, M. J. (1973). Schedule induced behavior in humans. Physiology \& Behavior, 11, 395-398.

Porter, J. G., \& Bryant, W. E., Jr. (1978). Adjunctive behavior in the Mongolian gerbil. Physiology \& Behavior, 16, 151-155.

Porter, J. G., \& Kenshalo, D. R. (1974). Schedule-induced drinking following omission of reinforcement in the rhesus monkey. Physiology \& Behavior, 12, 1075-1077.

SEGAL, E. F. (1965). The development of water drinking on a dry food free-reinforcement schedule. Psychonomic Science, 2, 29-30.

Shanab, M. E., \& Peterson, J. L. (1969). Polydipsia in the pigeon. Psychonomic Science, 15, 51-52.

Staddon, J. E. R. (1977). Schedule-induced behavior. In W. K. Honig \& J. E. R. Staddon (Eds.), Handbook of operant behavior. Englewood Cliffs, NJ: Prentice-Hall.

(Manuscript received for publication May 21, 1984.) 